\title{
Investigating the time-scaling behavior of the 2004-2010 seismicity of Aswan area (Egypt) by means of the Allan factor statistics and the detrended fluctuation analysis
}

\author{
L. Telesca ${ }^{1}$, M. Lovallo ${ }^{2}$, A. E.-E. Amin Mohamed $^{3}$, M. ElGabry ${ }^{3}$, S. El-hady ${ }^{3}$, K. M. Abou Elenean ${ }^{3}$, and R. ElShafey \\ Fat ElBary ${ }^{4}$ \\ ${ }^{1}$ Consiglio Nazionale delle Ricerche, Istituto di Metodologie per l'Analisi Ambientale, C.da S.Loja, 85050 Tito (PZ), Italy \\ ${ }^{2}$ Agenzia Regionale per la Protezione dell'Ambiente di Basilicata, 85100 Potenza, Italy \\ ${ }^{3}$ National Research Institute of Astronomy and Geophysics NRIAG, 11421 Helwan, Cairo, Egypt \\ ${ }^{4}$ Aswan Seismological Research Institute, Egypt
}

Correspondence to: L. Telesca (luciano.telesca@imaa.cnr.it)

Received: 10 October 2011 - Accepted: 2 November 2011 - Published: 4 May 2012

\begin{abstract}
The time dynamics of seismicity of Aswan area (Egypt) from 2004 to 2010 was investigated by means of the (i) Allan Factor, which is a powerful tool allowing the capture of time-clusterized properties of temporal point processes; and the (ii) detrended fluctuation analysis, which is capable of detecting scaling in nonstationary time series. The analysis was performed varying the depth and the magnitude thresholds. The 2004-2010 Aswan seismicity is characterized by significant three-fold time-clustering behaviors with scaling exponents $\sim 0.77$ for timescales between $10^{4.16} \mathrm{~s}$ and $10^{5.14} \mathrm{~s}, \sim 0.34$ for timescales between $10^{5.14} \mathrm{~s}$ and $10^{6.53} \mathrm{~s}$, and $\sim 1$ for higher timescales. The seismic interevent times and distances are characterized by persistent temporal fluctuations for most of the magnitude and depth thresholds.
\end{abstract}

\section{Introduction}

A marked temporal stochastic point process describes events occurring randomly in time (Cox and Isham, 1980) marked by the intensity of the events, and is completely defined by the set of the time occurrences. Such representation was used in modelling several and diverse point processes, like earthquakes (Telesca and Lovallo, 2009; Telesca et al., 2009a, b), lightning (Telesca et al., 2005), starquakes (Telesca, 2005), solar flares (Telesca, 2007), and also some human and social disasters (Telesca and Lovallo, 2006).

A fractal point process displays power-law form in several of its relevant statistics with related scaling exponents, indicating that the represented phenomen contains clusters of points over a relatively large set of timescales (Lowen and Teich, 1995). A fractal point process is, then, characterized by time-clustering behavior, contrarily to homogeneous Poissonian processes, whose density of the event occurrences is nearly constant through time. Generally, in order to capture the main characteristics of the time dynamics of a process, the power spectral density (PSD) is the first method to be used because it furnishes information on the frequency distribution of the process power, which is the physical quantity characterizing a process. By using the Fourier transform, the PSD can be calculated by means of the coefficients of the Fourier transform. The PSD allows detection of periodic or scaling behavior. Periodicities can be revealed by spike-like variations in the PSD, while a power-law shape $f^{-\alpha}$ reveals that the process is scaling; the power-law exponent, also called the scaling exponent, conveys qualitative and quantitative information about the type and strength of the temporal fluctuations governing the process. If the scaling exponent is approximately zero for a wide range of frequency bands, the PSD is approximately flat and the process is a realization of a white noise process, characterized by completely random fluctuations, independence and uncorrelation among all its values as well as absence of any kind of memory phenomenon. If the scaling exponent is negative, the PSD behaves as an increasing function of the frequency $f$; this indicates that the high-frequency temporal fluctuations are predominant. In this case the process is negatively correlated or antipersistent. The antipersistence is a dynamical property of a process in which an increase (decrease) of the process in one period is very likely followed by a decrease 
(increase) in the next period. The process appears very alternating and very irregular. If the scaling exponent is positive, the PSD behaves as a decreasing function of the frequency $f$; this indicates that the low-frequency temporal fluctuations are predominant, and the process appears smoother than the white noise. In this case the process is positively correlated or persistent. The persistence characterizes those processes in which an increase (decrease) of the process in one period is very likely followed by a increase (decrease) in the next period. The process appears very regular. Antipersistent processes are governed by feedback negative mechanisms, while persistent processes by positive ones (Cuomo et al., 2000).

It is clear from the above description that the determination of the spectral characteristics of a process allows one to know deeply about the underlying governing mechanism. If the calculation of the PSD is straightforward for a timecontinuous process by means of the Fourier transform, the evaluation of the spectral properties of a point process, like an earthquake sequence, cannot be directly performed; yet such evaluation is important if one wants to get more insight into its dynamics.

Therefore, appropriate statistical methods have to be applied, and first of all appropriate descriptions of the point process have to be considered. A point process can be represented in two ways: (1) using the interevent interval series, or (2) forming its relative counting process. In the first representation, the time series is formed by the rule $T_{i}=t_{i+1}-t_{i}$, where $t_{i}$ indicates the occurrence time numbered by the index $i$. In the second representation, the time axis is divided into equally spaced contiguous counting windows of duration $\tau$ to produce a sequence of counts $\left\{N_{k}(\tau)\right\}$, where $N_{k}(\tau)$ represents the number of events falling into the $k$-th window of duration $\tau$. The duration $\tau$ of the window is called counting time or timescale. The latter approach considers the earthquakes as the events of interest and assumes that there is an objective clock for the timing of the events. The former approach emphasizes the interevent intervals and uses the event number as an index of the time.

Both representations allow us to apply several statistical techniques to an earthquake sequence. In particular, in this study we will use the Allan Factor (AF) to analyze the time dynamics of earthquakes represented by counting processes, and we will then use the detrended fluctuation analysis to analyze the seismic interevent intervals and interdistances of one of the most interesting reservoirs in the world, the Aswan area, where reservoir-induced as well as tectonic earthquakes have occurred. The scientific and practical importance of investigating possible mechanisms related to the dynamics of influence of high dam water reservoirs on local earthquakes generation is very challenging. Since the middle of the past century, reservoir-induced seismicity has been observed at many reservoirs and their geological, hydrological and physical features are still subjects of intense investigations (DuráGómez and Talwani, 2010). Dynamical aspects of the seismic problem, along with the application of advanced time se- ries tools, are less investigated (Peinke et al., PEPI, 156/1-2, 130-142, 2006; Matcharashvili et al. 2008; Telesca, BSSA, 2010).

\section{The Aswan area}

We analyzed the seismicity of the Aswan area (Egypt), which represents one of the most interesting seismic areas in the world due to the possible reservoir-related earthquake triggering mechanisms linked with the loading/unloading operations of the Lake Nasser (Fig. 1). The data from 5 October 2004 to 31 December 2010 were extracted from the Bulletin of the Egyptian National Seismological Network (ENSN), compiled by the National Research Institute of Astronomy and Geophysics, Helwan, Cairo (Egypt) (4189 events).

Aswan hosts the largest dam in Egypt, "The high Dam", which is $111 \mathrm{~m}$ high, a crest length of $3830 \mathrm{~m}$ and a volume of 44300000 cubic meters, impounds a reservoir, Lake Nasser, that has a gross capacity of 169 billion cubic meters. Of the Nile's total annual discharge, some 74 billion cubic meters of water have been allocated by treaty between Egypt and The Sudan, with about 55.5 billion cubic meters apportioned to Egypt and the remainder to The Sudan. Lake Nasser backs up the Nile about $320 \mathrm{~km}$ in Egypt and almost $160 \mathrm{~km}$ farther upstream (south) in the Sudan (Latif, 1984), making the high dam one of the largest dams in the world and Nasser lake one of the largest reservoirs in the world as well.

Aswan area is known to be seismically active since the occurrence of the November 1981 Aswan earthquake $\left(M_{\mathrm{w}}=\right.$ 5.8). Detailed geological and geophysical surveys in the area confirm the existence of a few active faults to the southwest of the Aswan High Dam (e.g. Kalabsha, Khor El-Ramla and Kurkur faults) (Woodward-Clyde Consultants, 1985; Abou Elenean, 1997; Abou Elenean, 2003). The seismic activity in this area might be related to both tectonic activities along these active faults and/or reservoir-induced seismicity due to the Nasser Lake.

Regional seismological network was established in 1982 around the northern part of the Naser Lake to monitor earthquake activity. Seismological studies have shown that the majority of the local earthquakes appear to be concentrated at the intersection of the E-W and the N-S faults. This intersection is considered a convenient location for stress accumulation (Talwani, 1989). Generally, the overall faulting displacement in this zone is strike-slip with small normal component. The N-S faults have a low degree of seismic activity compared with the E-W faults. Woodword-Clyde Consultants (1985) related the existence of the E-W faults to small differential spreading rates in the northern Red Sea to the north and south of a zone between $22^{\circ}$ and $24^{\circ}$ north. 


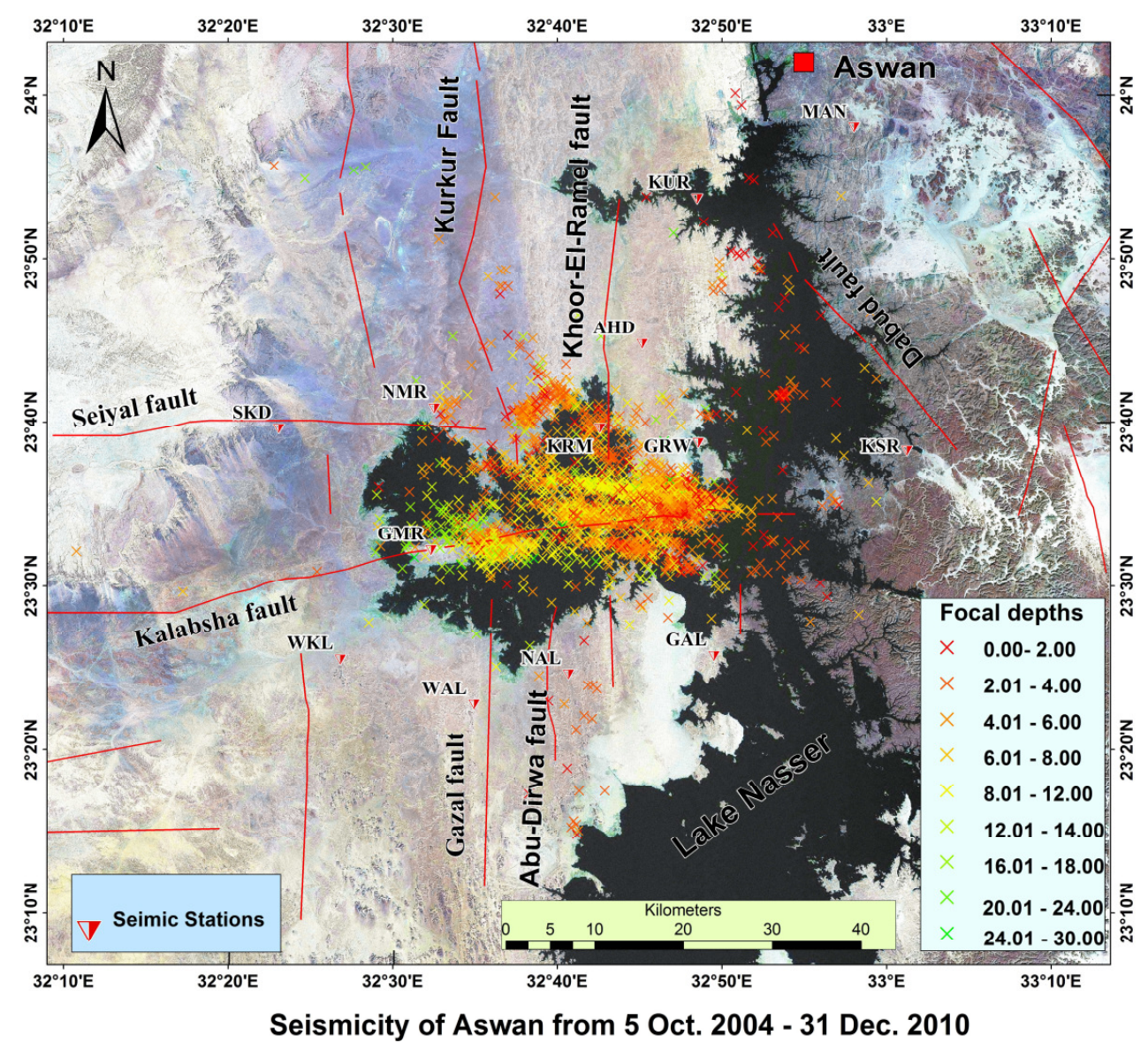

Fig. 1. Seismicity of the Aswan area (Egypt) from 2004 to 2010.

\section{Methods}

\subsection{The Allan Factor}

The Allan Factor (AF) (Allan, 1966) is related to the variability of successive counts, useful to detect the event clustering in a point process. Dividing the whole observation period in nonoverlapping windows of duration $\tau$, called timescale, we count the number of events falling in each k-th window and we form the counting process $\mathrm{N}_{k}(\tau)$. The AF is defined as the variance of successive counts for a specified counting time $\tau$ divided by twice the mean number of events in that counting time

$\mathrm{AF}(\tau)=\frac{<\left(N_{k+1}^{(\tau)}-N_{k}(\tau)\right)^{2}>}{2<N_{k}(\tau)>}$.

This measure reduces the effect of possible nonstationarity of the point process, because it is defined in terms of the difference of successive counts (Viswanathan et al., 1997). Varying the timescale $\tau$ allows producing a relationship between $\operatorname{AF}(\tau)$ and $\tau$, useful to detect scaling behavior in the sequence.
The AF of a fractal point process varies with the counting time $\tau$ with the power-law form:

$\operatorname{AF}(\tau)=1+\left(\frac{\tau}{\tau_{1}}\right)^{\alpha}$

with $0<\alpha<3$ over a large range of counting times $\tau$ (Lowen and Teich, 1995). $\tau_{1}$ is the so called fractal onset time for the $\mathrm{AF}$ and is estimated as the crossover timescale between the Poissonian (where AF assumes values near unity) and scaling behavior.

\subsection{The Detrended Fluctuation Analysis}

The detrended fluctuation analysis (DFA) was proposed by Peng et al. (Peng et al., 1995), and it avoids spurious detection of correlations that are artifacts of nonstationarity that often affects experimental data. The methodology operates on the time series $x(i)$, where $i=1,2, \ldots, N$ and $N$ is the length of the series. With $x_{\text {ave }}$ we indicate the mean value

$x_{\mathrm{ave}}=\frac{1}{N} \sum_{k=1}^{N} x(k)$. 
The signal is first integrated

$y(k)=\sum_{i=1}^{k}\left[x(i)-x_{\mathrm{ave}}\right]$.

Next, the integrated time series is divided into boxes of equal length, $n$. In each box a least-squares line is fitted to the data, representing the trend in that box. The $y$ coordinate of the straight line segments is denoted by $y_{n}(k)$. Next we detrend the integrated time series $y(k)$ by subtracting the local trend $y_{n}(k)$ in each box. The root-mean-square fluctuation of this integrated and detrended time series is calculated by

$F(n)=\sqrt{\frac{1}{N} \sum_{k=1}^{N}\left[y(k)-y_{n}(k)\right]^{2}}$.

Repeating this calculation over all box sizes, we obtain a relationship between $F(n)$, that represents the average fluctuation as a function of box size, and the box size n. If $F(n)$ behaves as a power-law function of $n$, data present scaling:

$F(n) \propto n^{d}$.

Under these conditions the fluctuations can be described by the scaling exponent $d$, representing the slope of the line fitting $\log [F(n)]$ to $\log (n)$. For a white noise process, $d=0.5$. $0.5<d<1.0$ indicates the presence of persistent long-range correlations, meaning that a large (compared to the average) value is more likely to be followed by a large value and vice versa. $0<d<0.5$ indicates the presence of antipersistent long-range correlations, meaning that a large (compared to the average) value is more likely to be followed by a small value and vice versa.

\section{Results}

The analysis was performed on the seismic sequences with magnitude $\mathrm{m} \geq 1.2$ and depth $\mathrm{h} \leq 15 \mathrm{~km}$. The lower limit for the magnitude represents the completeness magnitude of the series during the investigated period.

We applied the Allan Factor (AF) method to identify and quantify time-clustering in the sequence of the earthquakes. Figure 2 shows the $\mathrm{AF}$ of the seismic sequences recorded in the Aswan area for timescales $\tau$ from $10 \mathrm{~s}$ to about $3 \mathrm{yr}$; the upper timescale approximately corresponds to the $1 / 10$ of the entire period (higher timescales would lead to misleading results for the poorer statistics). Starting from $\tau_{1}>10^{4.16} \mathrm{~s}(\sim 4 \mathrm{~h})$, the AF curve reveals visible power-law behavior. In particular, the AF curve is characterized by three different timescale regimes, with three different scaling exponents, separated by three different crossover timescales: $\tau_{1}=10^{4.16} \mathrm{~s}, \tau_{2}=10^{5.14} \mathrm{~s}(\sim 1.6$ days $)$ and $\tau_{3}=10^{6.53} \mathrm{~s}(\sim 40$ days $)$. From $\tau_{1}$ to $\tau_{2}$ the AF increases with a power-law with scaling exponent $\alpha_{1}=0.77 \pm 0.04$; between $\tau_{2}$ and $\tau_{3}$ with scaling exponent $\alpha_{2}=0.34 \pm 0.05$,

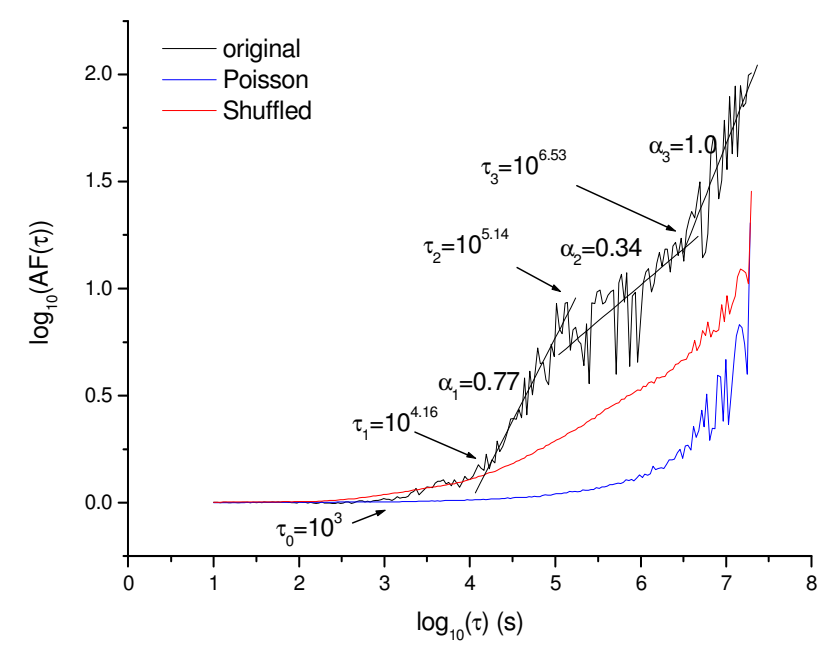

Fig. 2. AF of the seismic sequence recorded in the Aswan area plotted in Fig. 1 and for $\mathrm{m} \geq 1.2$ and depth $\mathrm{h} \leq 15 \mathrm{~km}$. The blue curve is the Poissonian $95 \%$ confidence and the red curve is the random shuffling $95 \%$ confidence.

and for timescales higher than $\tau_{3}$ with scaling exponent $\alpha_{3}=1.0 \pm 0.1$. The cutoff timescale $10^{4.16} \mathrm{~s}$ can be considered an estimation of the so-called fractal onset time (Thurner et al., 1997) that indicates the lower timescale from which the clustering behavior can be detected and quantified. The early flatness up to about $10^{3} \mathrm{~s}$ indicates a Poissonian-like behavior of the sequence for small timescales. The intermediate timescale region between $10^{3} \mathrm{~s}$ and $10^{4.16} \mathrm{~s}$ can be considered as a "transfer" timescale region between the two opposite behaviors, from Poissonian to clusterized dynamics. In all the three regimes, the value of the scaling exponent indicates a clustering behavior of the sequence, which is stronger at higher timescales and weaker at intermediate timescales. In order to check the significance of such behavior and verify that the obtained AF curve is significantly distinguished from that obtained by a Poissonian process, we generated 1000 Poissonian sequences, characterized by identical mean intervent time and identical number of events of the orginal sequence. To each simulated sequence the AF was applied. For each timescale the 95th percentile among the AF values for that timescale was calculated. The final $95 \%$ confidence AF curve was then given by the set of the 95th percentiles (blue curve in Fig. 2). The AF curve is significantly different from those obtained by the Poisson surrogates within the scaling range; therefore, the scaling behavior of the seismic cluster is significantly non-Poissonian. In order to check whether the scaling behavior of the sequence is due to the shape of the probability density function of the interevent times or to the their orderings, we shuffled 1000 times the interevent intervals, and for each shuffle we calculated the AF curve. The $95 \%$ confidence AF curve for the shuffles was calculated as above. Such curve (red curve in Fig. 2) is lower than the AF 


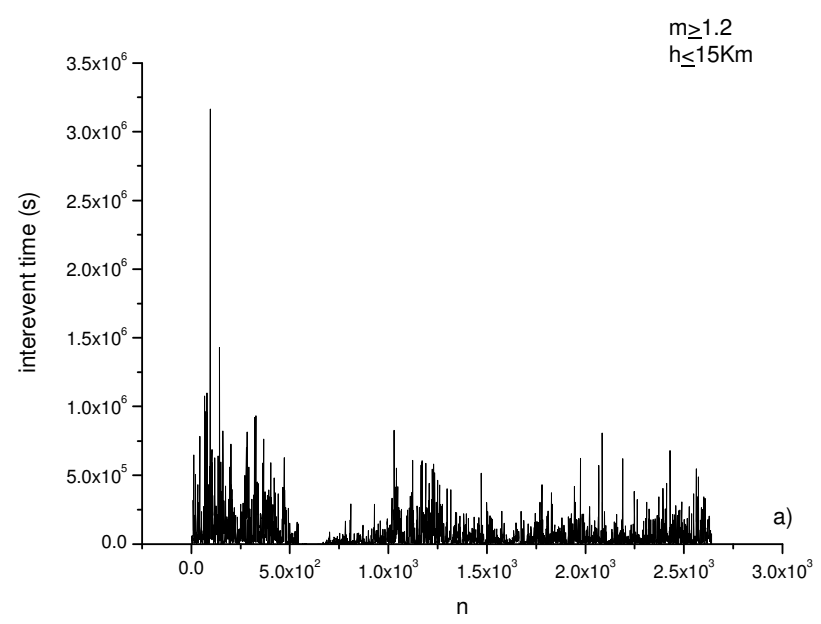

Fig. 3. Interevent time series of the sequence of the seismic events with magnitude $\mathrm{m} \geq 1.2$ and depth $\mathrm{h} \leq 15 \mathrm{~km}$.

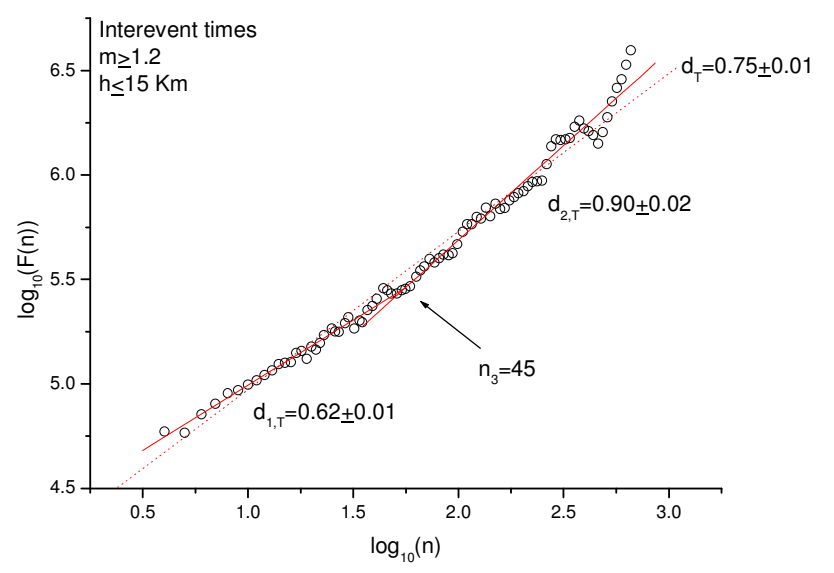

Fig. 4. DFA results for the interevent time series shown in Fig. 6.

curve of the original sequence within the scaling range, and this indicates that the scaling behavior is due to the specific ordering of the interevent intervals.

We applied the DFA to analyze the persistent properties of the seismic interevent times. Figure 3 shows, as an example, the interevent (in seconds) time series of the sequence of the seismic events with magnitude $\mathrm{m} \geq 1.2$ and depth $\mathrm{h} \leq 15 \mathrm{~km}$. The AF and the DFA describe the time properties of the seismic sequences, and are complementary statistical approaches (Viswanathan et al., 1997). The first treats the earthquakes as a point process, while the second treats it as a sequence of interevent intervals; also, the scaling regions identified in the $\mathrm{AF}$ and DFA curves cannot be matched exactly since the AF is referenced to real time, while the DFA function is referenced to interval number (Viswanathan et al., 1997). Nevertheless, applying the relationship between interval-based frequency (in cycles/interval) and the time-based frequency (in cycles/s) (Teich et al., 2001)

$f_{\tau}=\frac{f_{n}}{<T>}$,

where $f_{\tau}$ indicates the time-based frequency, $f_{n}$ indicates the interval-based frequency and $\langle T\rangle$ the mean interevent time, we can find the relationship between the timescale $\tau$ in the AF statistics with the scale $n$ in the DFA method as

$n=\frac{\tau}{<T>}$.

Applying Eq. (8), the crossover timescales identified in the AF curve of Fig. 3 can be converted in event numbers; in particular, $\tau_{2} \sim 10^{5.14} \mathrm{~s}$ corresponds to $n_{2} \sim 2$ and $\tau_{3} \sim 10^{6.53} \mathrm{~s}$ corresponds to $n_{3} \sim 45$.

Figure 4 shows the DFA results for the interevent time series shown in Fig. 3 for scales between 4 and $N / 4$, where $N$ is the length of the interevent time series. We fitted the fluctuation curve in the two scaling regions: the first between 4 and 45 , the second between 45 and $N / 4$. These two scaling ranges correspond approximately to the second and the third timescale regions identified in the AF curve of Fig. 3. The scaling exponent in the first scaling region is $\sim 0.62$ and the one in the second scaling region is $\sim 0.90$, indicating persistence. Using the relationship between the time-base spectral exponent $\alpha$ and the DFA scaling exponent $d$ (Buldyrev et al., 1995)

$d=\frac{1+\alpha}{2}$,

the scaling exponents estimated in the AF curve are consistent with those estimated in the DFA curve. From Eq. (9), we derive $d_{1}=0.67$ and $d_{2}=1.0$, which are close to the values estimated from the DFA curve. The DFA does not allow identification of the first AF timescale region (from $\tau_{1} \sim 10^{4.16} \mathrm{~s}$ to $\tau_{2} \sim 10^{5.14} \mathrm{~s}$ ), which is probably linked with the swarm-like behavior of the seismicity at almost the middle of the observation period. The presence of different scaling regions in the DFA was already found in Chen et al. (2002), who analysed the effects on the DFA curve of the superposition of different dynamics with distinct correlations. It should be observed that due to Eq. (9), the range $[0,1]$ of the scaling exponents estimated by the AF method corresponds to the range $[0.5,1]$ of the scaling exponents estimated by the DFA method; this should imply a better discrimination of the scaling regions performed by the AF method. However, when fitting the whole fluctuation function (dotted red line in Fig. 4), we get the scaling exponent $\mathrm{d}_{T} \approx 0.75$, which is approximately an average between the two scaling exponents $\mathrm{d}_{T, 1}$ and $\mathrm{d}_{T, 2}$; therefore, we can also use the slope of the line fitting the whole fluctuation function to describe the average scaling behavior of the sequence.

In order to investigate the variation of the time-clustering behavior with the threshold magnitude and threshold depth, we applied the AF to the seismic sequences, varying the 


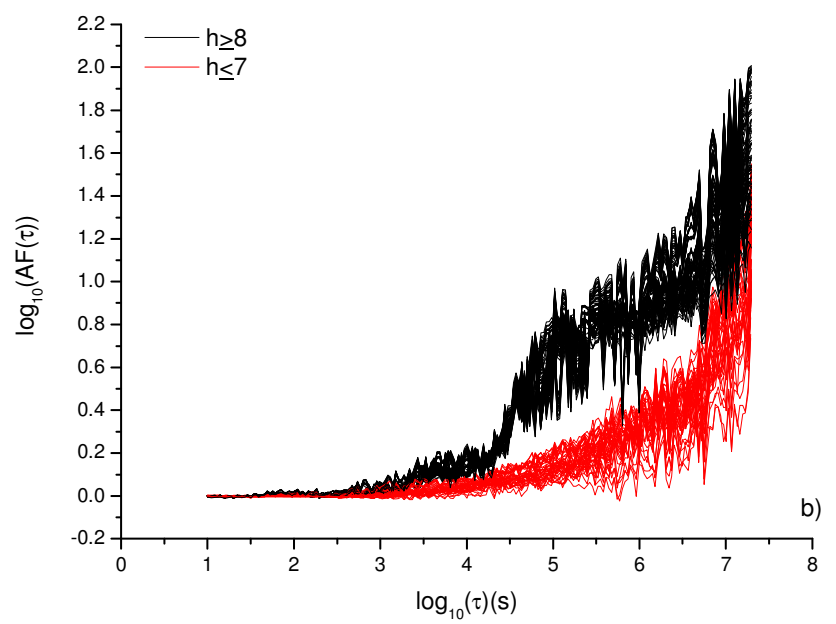

Fig. 5. AF curves for changing threshold magnitude and depth.
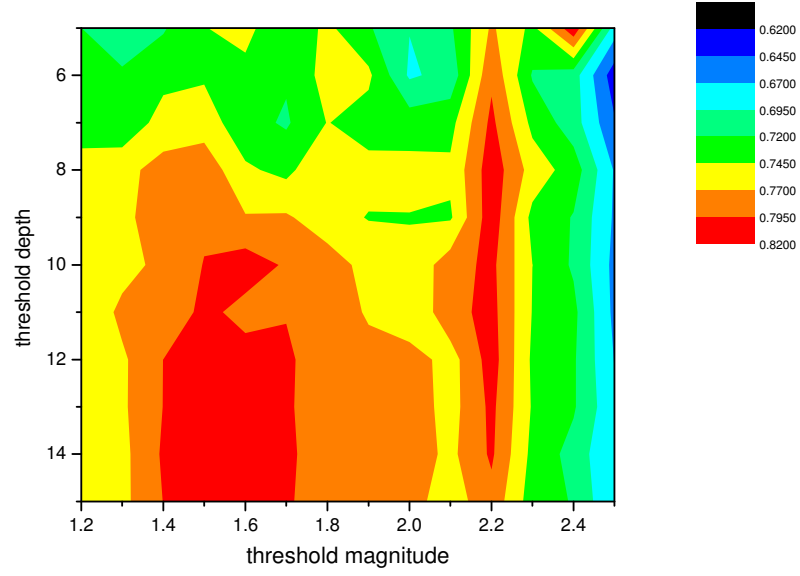

Fig. 6. Scaling exponents $d_{T}$ estimated by performing the DFA over the interevent time series changing the threshold magnitude and the threshold depth.

threshold magnitude from 1.2 to 2.5 with 0.1 step and the threshold depth from $5 \mathrm{~km}$ to $15 \mathrm{~km}$ with $1 \mathrm{~km}$ step, with timescale ranging from $10 \mathrm{~s}$ to $1 / 10$ of the whole period of each sequence. The results are shown in Fig. 5, and two distinct patterns are clearly evidenced, depending on the threshold depth: for threshold depths $\mathrm{h} \leq 7 \mathrm{~km}$ the AF curves (red in Fig. 5) show a Poissonian behavior; while for threshold depths $\mathrm{h} \geq 8 \mathrm{~km}$ the AF curves (black in Fig. 5) show approximately three scaling regions, similar to the AF curve shown in Fig. 2. Such two-fold pattern does not depend on the threshold magnitude but only on the threshold depth. This indicates that shallower events tend to follow a Poissonian dynamics.

We performed the DFA on the interevent time series, varying the threshold magnitude from 1.2 to 2.5 with 0.1 step and the threshold depth from $5 \mathrm{~km}$ to $15 \mathrm{~km}$ with $1 \mathrm{~km}$ step. For all the series the fluctuation function was calculated in the timescale range between 4 and $N / 4$, where $N$ is the length of the series, and the scaling exponents were calculated as the slope of the line fitting the whole fluctuation function. Figure 6 shows the scaling exponents $\mathrm{d}_{T}$ changing the threshold magnitude and the threshold depth; it is clearly visible that for any threshold the series are persistent in time. In order to evaluate the significance of the estimates of the exponents, we generated 1000 shuffled series of any original sequence, randomly permutating the interevent time series, as outlined above (Telesca et al., 2003). Our aim was to test whether the calculated estimates of the scaling exponents $\mathrm{d}_{T}$ indicate a significant correlated effect with respect to the uncorrelated behavior displayed by the surrogates. Being $\mathrm{d}_{T}$ is the scaling exponent of the original sequence, let $\mu_{T}$ and $\sigma_{T}$ indicate the mean and the standard deviation of the scaling exponents calculated for the shuffled sequences of the interevent. We define the significance of our measure by the difference between the original and the mean surrogate value of the scaling exponents, divided by the standard deviation of the surrogate values (Theiler et al., 1992):

$\sigma_{T}=\frac{\left|d_{T}-\mu_{T}\right|}{\sigma_{T}}$.

Then the p-value is calculated by means of the formula $\mathrm{p}_{T}=\operatorname{erfc}\left(\sigma_{T} / \sqrt{ } 2\right)$ (Theiler et al., 1992); this is the probability of observing a significance $\sigma_{T}$ or larger if the null hypothesis (absence of any correlation) is true. Figure 7 shows the $\mathrm{p}$-values for the interevent time series changing the threshold magnitude and depth; the higher p-values (indicating statistical similarity with the shuffles) generally are for larger threshold magnitudes and smaller threshold depths. This indicates that the scaling exponents $\mathrm{d}_{T}$ are significantly higher than those obtained by the surrogate uncorrelated sequences for lower threshold magnitudes and higher threshold depths; this implies that more intense and shallower events tend to behave more likely as uncorrelated processes. This result is in good agreement with those obtained applying the AF method with changing threshold magnitude and depth.

We analysed the interdistance time series, which is the time series of the mutual distance between successive events, in order to investigate the temporal properties of the space distribution of the seismicity. Figure 8 shows, as an example, the interdistance time series, measured in degrees of arc length, of the sequence of the seismic events with magnitude $\mathrm{m} \geq 1.2$ and depth $\mathrm{h} \leq 15 \mathrm{~km}$. Figure 9 shows the DFA results for the interdistance time series shown in Fig. 9. The series is persistent with scaling exponents $\mathrm{d}_{S}$ approximately 0.75 . This indicates that the seismic events tends to clusterize not only in time but also in space. The DFA was applied to the interdistance time series, varying the threshold magnitude from 1.2 to 2.5 with 0.1 step, and the threshold depth from $5 \mathrm{~km}$ to $15 \mathrm{~km}$ with $1 \mathrm{~km}$ step, similarly to the interevent time series. Figure 10 shows the scaling exponents $\mathrm{d}_{S}$ changing the threshold magnitude and the threshold 


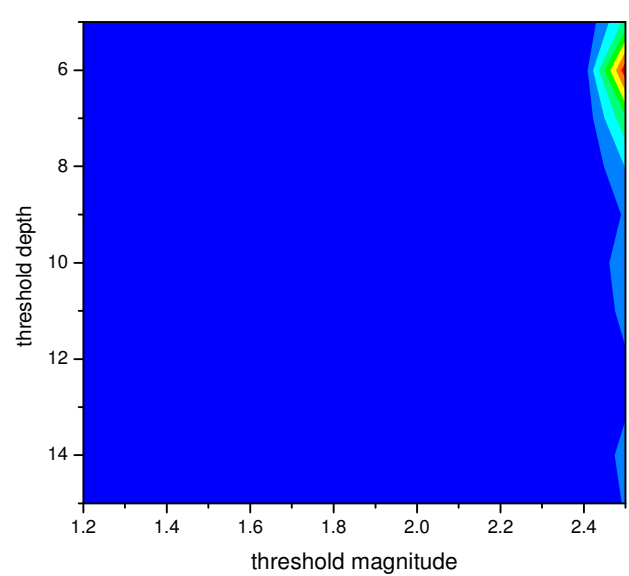

Fig. 7. p-values for the interevent time series changing the threshold magnitude and depth.

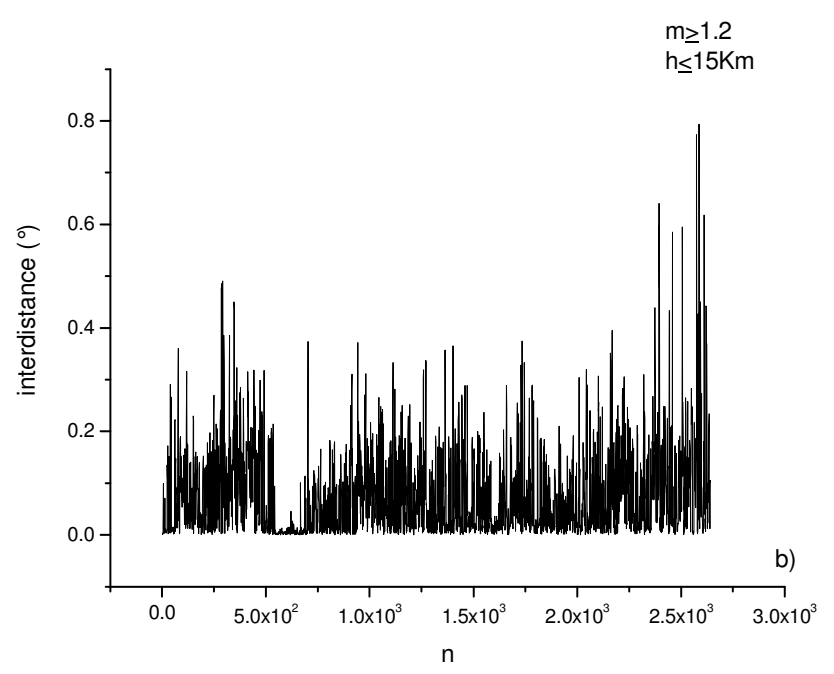

Fig. 8. Interdistance time series of the sequence of the seismic events with magnitude $\mathrm{m} \geq 1.2$ and depth $\mathrm{h} \leq 15 \mathrm{~km}$.

depth; it is clearly visible that for any threshold the series are persistent in space. We analysed the significance of the exponents $\mathrm{d}_{S}$, similarly to that evaluated for the scaling exponents $\mathrm{d}_{T}$, and Fig. 11 shows the p-values, which are generally higher for larger threshold magnitudes and smaller threshold depths. This indicates that the scaling exponents $\mathrm{d}_{S}$ are significantly higher than those obtained by the surrogate uncorrelated sequences for lower threshold magnitudes and higher threshold depths, suggesting a tendency to uncorrelation for more intense and shallower events.

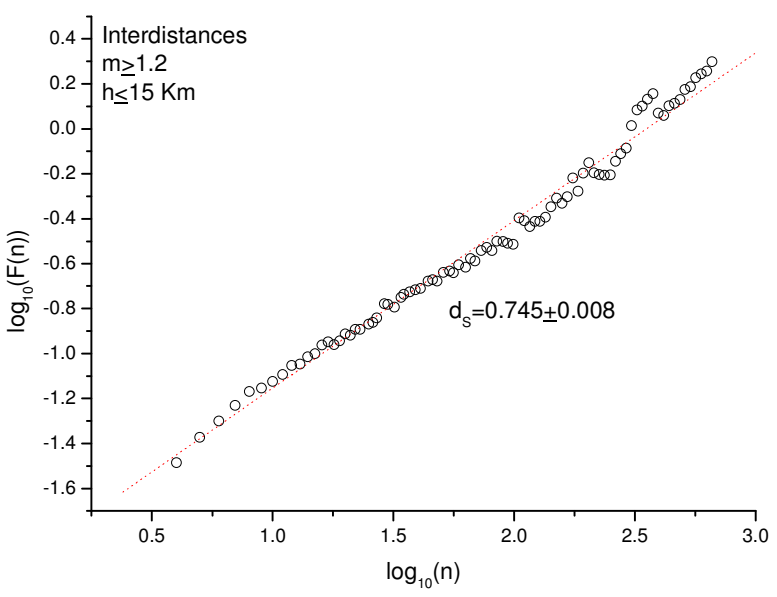

Fig. 9. DFA results for the interdistance time series shown in Fig. 13.

\section{Discussion}

Time-clustering of a point process can be described by the increase of occurrence frequency of events in a relatively narrow time interval against the general low occurrence frequency over a longer time span. Accordingly, it is considered as the opposite of temporal homogeneous Poissonian behavior, in which the rate of occurrence of the events is constant. The mechanisms responsible for time-clustering in earthquakes might be related to triggering by the occurrences of great or major earthquakes in neighboring segments, either to the transfer of co-seismic dynamic or static stress or to post-seismic relaxation of the stresses (Stein, 1999; Toda et al., 1998; Harris, 1998; Gomberg et al., 1998; Freed and Lin, 1998; McCloskey et al., 2005). However, in some cases the distance between two consecutive earthquakes is too large to indicate stress interaction amongst the earthquakes, and thus the time clustering of earthquakes could be due just to chance, in which earthquake occurrence is almost simultaneous in two or more segments despite differences in the earthquake cycle due to difference in the phase of strain accumulation, rheology, plate convergence rate, etc., in these segments (Ambikapathy and Gahalaut, 2011). This effect was also found in the analysis of the persistence of seismicity of Umbria-Marche (Italy) by using the DFA (Telesca et al., 2008). More than one scaling region, as we found in the analysis of the Aswan seismicity, was found in several other seismic areas worldwide. Generally, a two-fold timeclustering behavior indicates the superposition of two time dynamics, one related with the background seismicity (large timescales) and the other linked with the aftershocks (intermediate timescales) (Telesca et al., 2002). Similarly, the volcanic seismicity at Etna volcano, Sicily (Italy), has shown the $\mathrm{AF}$ curve characterized by two scaling regions, with scaling exponent 0.7 at intermediate timescales consistent 

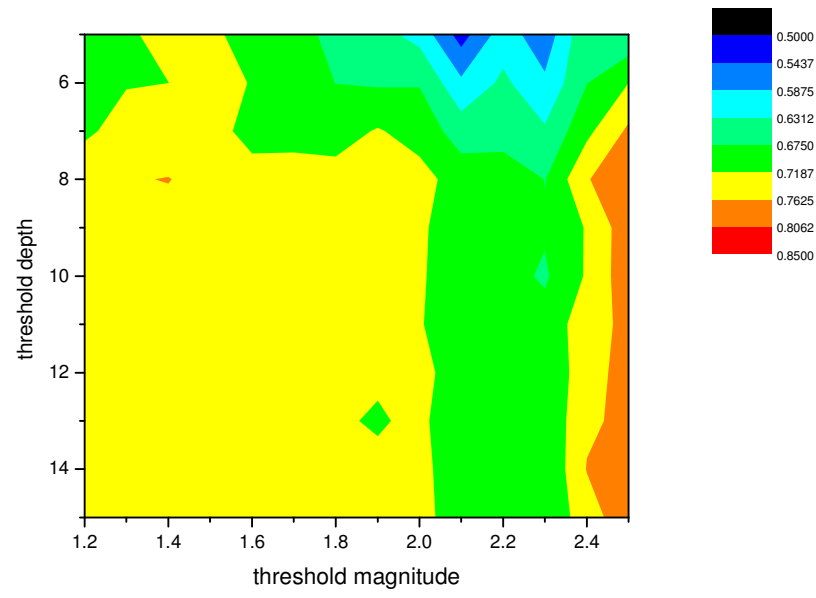

Fig. 10. Scaling exponents $d_{S}$ estimated by performing the DFA over the interdistance time series changing the threshold magnitude and the threshold depth.

with the timescales of the eruptive episodes, and 0.2 at large timescales describing the behavior of the background seismicity (Currenti et al., 2005). Single scaling region, in the AF or DFA curve, are shown by seismic series, in which the effect of the aftershocks is absent or negligible (Bohnestieh et al., 2003).

Time-clustering is a property that seems to characterize many natural processes. Forest fires are generally characterized by two-fold time-scaling behavior (mainly evident for lower threshold burned areas) with a crossover at a timescale of the order of days, separating the region of the lower timescales with scaling exponent smaller than that of the region of higher timescales (Telesca and Lasaponara, 2006). Two-fold time clustering behavior is also evidenced by lightning, but the scaling exponent for timescales lower than the crossover is larger than that for the higher timescales, revealing the intra-cluster (inside an individual thunderstorm) and inter-cluster (among successive thunderstorms) timecorrelation properties of lightning (Telesca et al., 2005). Using an event-like structure for the rain (Peters et al., 2002), where the rain events are defined as a sequence of non-zero rain rates and the size of a rain event is defined as the accumulated water column during the event, the presence of time-clustering dynamics for rain sequences with $\mathrm{AF}$ exponents around 0.3-0.4 was shown (Telesca et al., 2005). Scaling exponents ranging between 0.14 and 0.96 were found for 35 sequences of volcanic eruptions worldwide (Telesca et al., 2002). Very recently, the time-clustering characteristics in sequences of Italian landslides were analysed, revealing a scaling exponent ranging between 0.3 and 0.5 (Witt et al., 2010).

It is striking that many natural point processes are characterized by scaling behavior with an exponent not exceeding
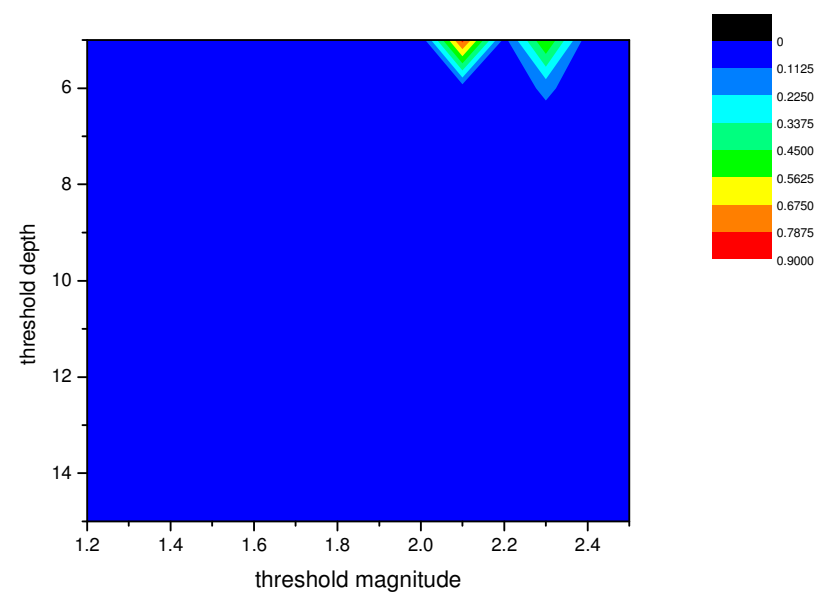

Fig. 11. p-values for the interdistance time series changing the threshold magnitude and depth.

1.5-2.0. This would suggest that there should be a kind of universality; and finding of the common dynamical characteristics in terms of time-clustering, correlations and persistence in all these diverse natural phenomena would be challenging.

\section{Conclusions}

The present paper deeply analyzes the time dynamics of the seismicity observed in the Aswan area (Egypt) between 2004 and 2010. The time dynamics of the seismicity, investigated by means of the Allan Factor, reveals a significant three-fold time-clustering behavior. The persistence of the interevent and the interdistance time series was analysed by means of the DFA. Our results point out significant correlated structures in the seismicity of the area, mainly for lower magnitude thresholds and higher depth thresholds.

Acknowledgements. The present study was supported by the CNR/ASRT 2011-2012 Project "Spatiotemporal seismicity patterns and earthquake precursors at Aswan, Egypt. Contribution to earthquake hazard assessment".

Edited by: M. E. Contadakis

Reviewed by: two anonymous referees

\section{References}

Abou Elenean, K. M.: Focal mechanism and stress tensor inversion of earthquakes in and around Nasser Lake, Egypt. Bull. Fac. of Sci. Zagazig Uni., Egypt. 25, 77-107, 2003.

Abou Elenean, K.: Seismotectonics of Egypt in relation to the Mediterranean and Red Seas tectonics, Ph. D. thesis Ain Shams Univ., Egypt, 191 pp., 1997.

Allan, D. W.: Statistics of atomic frequency standards, Proc. IEEE 54, 221-230, 1966. 
Ambikapathy, A. and Gahalaut, V. K.: Time clustering of earthquakes in the Sumatra-Andaman and Himalayan regions, Curr. Sci., 100, 1068-1071, 2011.

Bohnenstieh, D. R, Tolstoy, M., Smith, D. K., Fox, C. G., and Dziak, R. P.: Time-clustering behavior of spreading-center seismicity between 15 and $35^{\circ} \mathrm{N}$ on the Mid-Atlantic Ridge: observations from hydroacoustic monitoring, Phys. Earth Planet. Int. 138, 147-161, 2003.

Buldyrev, S. V., Goldberger, A. L., Havlin, S., Mantegna, R. N., Matsa, M. E., Peng, C.-K., Simons, M., and Stanley, H. E.: Longrange correlation properties of coding and noncoding DNA sequences: GenBank analysis, Phys. Rev. E. 51, 5084-5091, 1995.

Chen, Z., Ivanov, P. Ch., Hu, K., and Stanley, H. E.: Effect of nonstationarities on detrended fluctuation analysis, Phys. Rev. E, 65, 041107, 2002.

Cox, D. R. and Isham, V.: Point Processes, Chapman and Hall, London, 1980.

Cuomo, V., Lanfredi, M., Lapenna, V., Macchiato, M., Ragosta, M., and Telesca, L.: Antipersistent dynamics in short time scale variability of self-potentials, Ann. Geofis., 43, 271-278, 2000.

Currenti, G., Del Negro, C., Lapenna, V., Telesca, L.: Scaling characteristics of local geomagnetic field and seismicity at Etna volcano and their dynamics in relation to the eruptive activity, Earth Planet. Sci. Lett., 235, 96-106, 2005.

Durá-Gómez, I. and Talwani, P.: Reservoir-induced seismicity associated with the Itoiz Reservoir, Spain: a case study, Geophys. J. Int. 181, 343-356, 2010.

Freed, A. M. and Lin, J.: Time dependent changes in failure stress following thrust earthquakes. J. Geophys. Res. 103, 2439324409, 1998.

Gomberg, J., Beeler, N. M., Blanpied, M. L., and Bodin, P.: Earthquake triggering by transient and static deformations, J. Geophys. Res., 103, 24411-24416, 1998.

Harris, R.: Introduction to special section: stress riggers, stress shadows, and implications for seismic hazards, J. Geophys. Res., 103, 24347-24358, 1998.

Latif, A. F. A.: Lake Nasser The new man-made lake in Egypt (with reference to Lake Nubia), in: "Ecosystems of the World 23, Lakes and Reservoirs", edited by: F. B. Taub, 385-410, Elsevier Publishing Co., Amsterdam, Oxford, New York, Tokyo, 1984.

Lowen, S. B. and Teich, M. C.: Estimation and simulation of fractal stochastic point processes, Fractals, 3, 183-210, 1995.

Matcharashvili, T., Chelidze, T., and Peinke, J.: Increase of order in seismic processes around large reservoir induced by water level periodic variation, Nonlinear Dynam., 51, 399-407, 2008.

McCloskey, J., Nalbant, S. S., and Steacy, S.: Earthquake risk from co-seismic stress, Nature, 434, 291, 2005.

Peng, C.-K., Havlin, S., Stanley, H. E., and Goldberger, A. L.: Quantification of scaling exponents and crossover phenomena in nonstationary heartbeat time series, Chaos 5, 82-87, 1995.

Peters, O., Hertlein, C., and Christensen, K.: A complexity view of rainfall, Phys. Rev. Lett., 88, doi:10.1103/PhysRevLett.88.018701, 2002.

Peinke, J., Matcharashvili, T., Chelidze, T., Gogiashvili, J., Nawroth, A., Lursmanashvili, O., and Javakhishvili, Z.: Influence of Periodic Variations in Water Level on Regional Seismic Activity Around a Large Reservoir: Field and Laboratory Model, Phys. Earth Planet. Int., 156, 130-142, 2006.
Stein, S.: The role of stress transfers in earthquake occurrence, Nature, 402, 605-609, 1999.

Talwani, P.: Characteristic features of intraplate earthquakes, Seismol. Res. Lett., 59, 305-310, 1989.

Teich, M. C., Lowen, S. B., Jost, B. M., Vibe-Rheymer, K., and Heneghan, C.: Heart Rate Variability: Measures and Models, in: Nonlinear Biomedical Signal Processing, Vol. II, Dynamic Analysis and Modeling, edited by: M. Akay (IEEE Press, New York, 2001), 159-213, 2001.

Telesca, L.: Quantifying the time-clustering in SGR 1806-20 Bursts, Fluctuation Noise Lett., 5, L417-L422, 2005.

Telesca, L.: Identifying time-clustering structures in the sequence of solar flare hard X-ray bursts, Physica A, 384, 516-521, 2007.

Telesca, L.: Analysis of the cross-correlation between seismicity and water level in Koyna area (India), B. Seismol. Soc. Am., 100, 2317-2321, 2010.

Telesca, L. and Lasaponara, R.: Emergence of temporal regimes in fire sequences, Physica A, 359, 747-751, 2006.

Telesca, L. and Lovallo, M.: Are global terrorist attacks timecorrelated?, Physica A, 362, 480-484, 2006.

Telesca, L. and Lovallo, M.: Non-uniform scaling features in central Italy seismicity: a non-linear approach in investigating seismic patterns and detection of possible earthquake precursors, Geophys. Res. Lett., 36, L01308, doi:10.1029/2008GL036247, 2009.

Telesca, L., Bernardi, M., and Rovelli, C.: Intra-cluster and intercluster time correlations in lightning sequences, Physica A, 356, 655-661, 2005.

Telesca, L., Chen, C.-C., and Lee, Y.-T.: Scaling behaviour in temporal fluctuations of crustal seismicity in Taiwan, Nat. Hazards Earth Syst. Sci., 9, 2067-2071, doi:10.5194/nhess-9-2067-2009, 2009b.

Telesca, L., Colangelo, G., Lapenna, V., and Macchiato, M.: On the scaling behavior of rain event sequence recorded in Basilicata region (Southern Italy), J. Hydrology, 296, 234-240, 2005.

Telesca, L., Cuomo, V., Lapenna, V., and Macchiato, M.: Timeclustering analysis of volcanic occurrence sequences, Phys. Earth Planet. Int., 131, 47-62, 2002.

Telesca, L., Cuomo, V., Lapenna, V., and Macchiato, M.: 1/f $\alpha$ fluctuations of seismic sequences, Fluct. Noise Lett., 2, L357-L367, 2002.

Telesca, L., Lapenna, V., and Macchiato, M.: Spatial variability of time-correlated behaviour in Italian seismicity, Earth Planet. Sci. Lett., 212, 279-290, 2003.

Telesca, L., Lovallo, M., Lapenna, V., and Macchiato, M.: Spacemagnitude dependent scaling behaviour in seismic interevent series revealed by detrended fluctuation analysis, Physica A, 387, 3655-3659, 2008.

Telesca, L., Rouai, M., and Cherkaoui, T. E.: Time-clustering behavior in the sequence of the aftershocks of the AlHoceima (Morocco) 24 February 2004 earthquake, Nat. Hazards Earth Syst. Sci., 9, 2063-2066, doi:10.5194/nhess-9-2063-2009, 2009a.

Theiler, J., Eubank, S., Longtin, A., Galdrikian, B., and Farmer, J. D.: Testing for nonlinearity in time series: the method of surrogate data, Physica D, 58, 77-94, 1992.

Thurner, S., Lowen, S. B., Feurstein, M. C., Heneghan, C., Feichtinger, H. G., and Teich, M. C.: Analysis, Synthesis, and Estimation of Fractal-Rate Stochastic Point Processes, Fractals 5, 
565-596, 1997.

Toda, S., Stein, S., Reasenberg, P. A., Dieterich, J. H., and Yoshida, A.: Stress transferred by the $1995 \mathrm{Mw}=6.9$ Kobe, Japan, shocks: effect on aftershocks and future earthquake probabilities, J. Geophys. Res., 103, 24543-24565, 1998.

Viswanathan, G. M., Peng, C.-K., Stanley, H. E., and Goldberger, A. L.: Deviations from uniform power law scaling in nonstationary time series, Phys. Rev. E, 55, 845-849, 1997.
Witt, A., Malamud, B. D., Rossi, M., Guazzetti, F., and Peruccacci, S.: Temporal correlations and clustering of landslides, Earth Surf. Proc. Land., 35, 1138-1156, 2010.

Woodward-Clyde Consultants: Earthquake activity and stability evaluation for the Aswan High Dam, Unpublished report, High and Aswan Dam Authority, Ministry of Irrigation, Egypt, 1985. 